\title{
3D printing of mineral-polymer bone substitutes based on sodium alginate and calcium phosphate
}

\author{
Aleksey A. Egorov ${ }^{1}$, Alexander Yu. Fedotov ${ }^{1}$, Anton V. Mironov², Vladimir S. Komlev², \\ Vladimir K. Popov ${ }^{2}$ and Yury V. Zobkov ${ }^{1}$
}

\author{
Letter \\ Address: \\ ${ }^{1}$ A. A. Baikov Institute of Metallurgy and Materials Science, Russian \\ Academy of Sciences, Leninsky prospect 49, 119334, Moscow, \\ Russia and ${ }^{2}$ Institute of Photonic Technologies, Federal Scientific \\ Research Centre "Crystallography and Photonics", Russian Academy \\ of Sciences, 2 Pionerskaya St., 142092 Troitsk, Moscow, Russia \\ Email: \\ Vladimir S. Komlev* - komlev@mail.ru \\ * Corresponding author \\ Keywords: \\ 3D printing; bone graft; calcium phosphate; composite materials; \\ sodium alginate; tissue engineering
}

Beilstein J. Nanotechnol. 2016, 7, 1794-1799. doi:10.3762/bjnano.7.172

Received: 24 March 2016

Accepted: 03 November 2016

Published: 21 November 2016

This article is part of the Thematic Series "Biological and biomimetic materials and surfaces".

Guest Editor: S. N. Gorb

(C) 2016 Egorov et al.; licensee Beilstein-Institut. License and terms: see end of document.

\begin{abstract}
We demonstrate a relatively simple route for three-dimensional (3D) printing of complex-shaped biocompatible structures based on sodium alginate and calcium phosphate $(\mathrm{CP})$ for bone tissue engineering. The fabrication of $3 \mathrm{D}$ composite structures was performed through the synthesis of inorganic particles within a biopolymer macromolecular network during 3D printing process. The formation of a new CP phase was studied through X-ray diffraction, Fourier transform infrared spectroscopy and scanning electron microscopy. Both the phase composition and the diameter of the $\mathrm{CP}$ particles depend on the concentration of a liquid component (i.e., the "ink"). The 3D printed structures were fabricated and found to have large interconnected porous systems (mean diameter $\approx 800 \mu \mathrm{m}$ ) and were found to possess compressive strengths from 0.45 to $1.0 \mathrm{MPa}$. This new approach can be effectively applied for fabrication of biocompatible scaffolds for bone tissue engineering constructions.
\end{abstract}

\section{Introduction}

3D printing is one promising methodology for tissue engineering constructions with specific architectonics and properties. It has the attractive advantages of both accurate and reproducible layer-by-layer fabrication of complex-shaped structures [1-4]. A number of biocompatible materials, such as polymers of different nature (both natural and synthetic), as well as a

variety of calcium phosphates (CPs) are used for this purpose [2]. In this respect, the alginate-based materials are of particular interest. Alginate (extracellular polysaccharide) is a popular biomaterial because of a number of key advantages: convenient precursors, nontoxic, excellent biocompatibility and appropriate biodegradability [5-7]. Additionally, CPs are widely used 
for bone graft substitution due to their chemical affinity to the bone mineral content [8]. One of the strategies to improve the bioactivity of the polymer-based materials is to incorporate some inorganic phase, such as CP particles, into their structure [9-11]. The 3D printing of these materials is usually achieved by simple ink jet processing of a mechanical mixture of starting ingredients (mineral/polymer components), producing desirable structures directly through layer-by-layer manufacture of the desired product [10]. Therefore, the final 3D product consists of a mechanical mixture of the polymer slurry with more-or-less homogeneously distributed CP particles.

In this work we propose a new biomimetic approach in which $3 \mathrm{D}$ printing of composite structures involves a chemical interaction of the polymer slurry with a liquid "ink", leading to in situ formation of a CP phase in the final product. It is well known that alginate allows precipitation of inorganic phases within its macromolecular network [7] and this process provides inorganic phases with different crystals sizes and morphologies [7,12]. For all these reasons, 3D printing of bioinspired structures has gained considerable interest since its inception, primarily because it can lead to artificial bone grafts that are close to native bone. In our experiments the formation of a new inorganic phase during the $3 \mathrm{D}$ printing process was monitored by X-ray diffraction (XRD), Fourier transform infrared (FTIR) spectroscopy and scanning electron microscopy (SEM), enabling us to find optimal parameters for the developed route.

\section{Results and Discussion}

$3 \mathrm{D}$ printing is a powerful tool for the production of custom-designed and complex bone substitutes [2,3]. In our work, the fabrication of $3 \mathrm{D}$ composite structures was performed using a binary system based on aqueous solutions of sodium alginate containing $\mathrm{PO}_{4}{ }^{3-}$ groups. A calcium chloride aqueous solution, as a second component, was used as a source of $\mathrm{Ca}^{2+}$ ions. The $3 \mathrm{D}$ composite structures were fabricated in a cubic shape $\left(8 \times 8 \times 5 \mathrm{~mm}^{3}\right)$, comprising 30 interconnected longitudinal channels with dimensions of $\approx 800 \times 800 \mu \mathrm{m}^{2}$ running through the samples (Figure 1). This structure resulted in optimal pores sizes and good interconnectivity, which are of great importance for the design of $3 \mathrm{D}$ bone substitutes for biomedical applications.

The CP phase was formed upon mixing of the polymer slurry containing ammonium hydrogen phosphate with a calcium chloride aqueous solution during the printing process according to the following reaction:

$$
\left(\mathrm{NH}_{4}\right)_{2} \mathrm{HPO}_{4}+\mathrm{Ca}^{2+}+2 \mathrm{H}_{2} \mathrm{O} \rightarrow \mathrm{CaHPO}_{4} \cdot 2 \mathrm{H}_{2} \mathrm{O}+2 \mathrm{NH}_{4}
$$

In fact, the mixture of the phosphate source with alginate gives rise to a homogeneous gel, suggesting an interaction between the biopolymer and the $\mathrm{HPO}_{4}{ }^{2-}$. The stability and homogeneity of this gel can be explained by possible interactions between the $\mathrm{HPO}_{4}{ }^{2-}$ moiety and the carboxylate group of alginate. This physical bonding translates to mixtures that are rich in electronic pairs leading to a higher reactivity and mineralization potential that can be transformed into composite materials [13]. According to X-ray diffraction data, synthesis in the presence of glutamic acids in the reaction medium maintained at $\mathrm{pH}$ $4.5 \pm 0.5$ yields the formation of dicalcium phosphate dihydrate (DCPD) in the printed samples (Figure 2). When precipitation is performed at a higher $\mathrm{pH}$, as reported in $[7,14]$, the difference of electrical charges in alginate is greater. As a consequence, a more compact complex without compositional water is obtained. The formation of anhydrous dicalcium phosphate (monetite) would be then kinetically favored. The estimated average mineral contents are 90,70 and $54 \%$ of DCPD for alginate concentrations of $0.25,1.0$ and $2.0 \mathrm{wt} \%$, respectively.

Figure 3 shows SEM images of 3D printed samples, where inorganic matrix DCPD crystal is prepared with a $1 \mathrm{M}$ solution of ammonium hydrogen phosphate and alginate concentration of 0.25 wt \%. The morphology of the crystals show plate- and

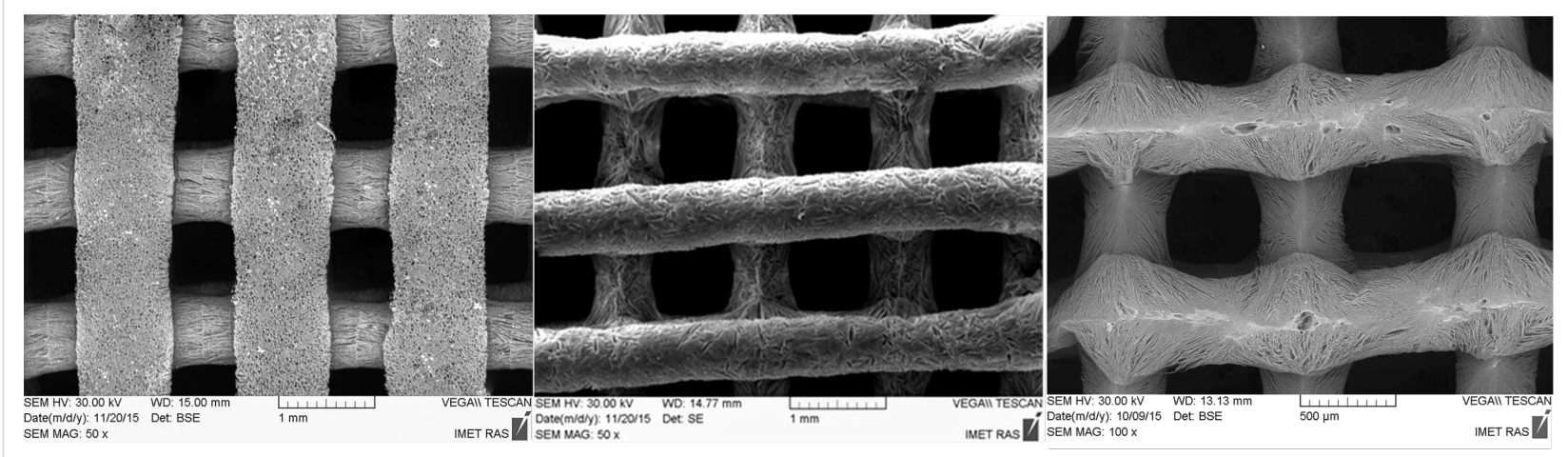

Figure 1: SEM micrographs of 3D printed samples. 


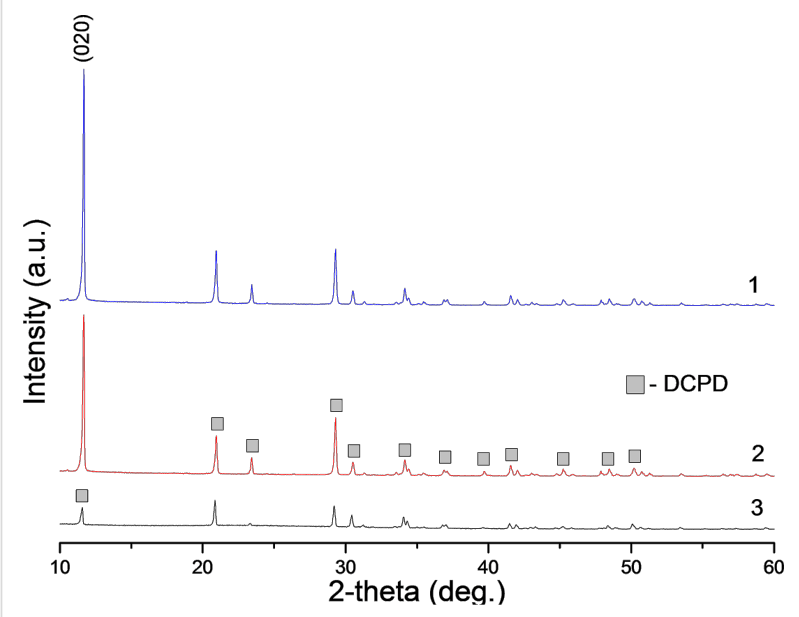

Figure 2: XRD spectra of 3D printed samples on the basis alginate with concentration (1) 0.25 wt \%, (2) 1.0 wt \% and (3) 2.0 wt \%. needle-like structures with different sizes. The formation of DCPD crystals is observed on the surface and internal regions of the 3D printed samples (Figure 3). Moreover, the SEM images demonstrate a decrease of plate-shaped DCPD particle for sizes from $\approx 40$ to $1 \mu \mathrm{m}$ with increasing alginate concentration from $0.25 \mathrm{wt} \%$ up to $2.0 \mathrm{wt} \%$ (Figure 4). This effect may be caused by an increase in concentration of heterogeneous nucleation centers of a calcium phosphate phase on the carboxyl groups of the amino acids. X-ray diffraction data lend support to this tendency: the height of the strongest peak (020) of DCPD decreases by a factor of 7 as the alginate and, correspondently, amino acid concentration increases from 0.25 to 2.0 wt \% (Figure 2).

The FTIR spectra of DCPD exhibits principal characteristic bands known to be associated with $\mathrm{PO}_{4}\left(v_{4}\right)$ at 575 and $1125 \mathrm{~cm}^{-1},\left(v_{1}\right)$ at $981 \mathrm{~cm}^{-1},\left(v_{3}\right)$ at $870 \mathrm{~cm}^{-1}$ and $1056 \mathrm{~cm}^{-1}$; as well as $\mathrm{H}_{2} \mathrm{O}$ at $650 \mathrm{~cm}^{-1}, 790 \mathrm{~cm}^{-1}$ [15]. In our FTIR spec-

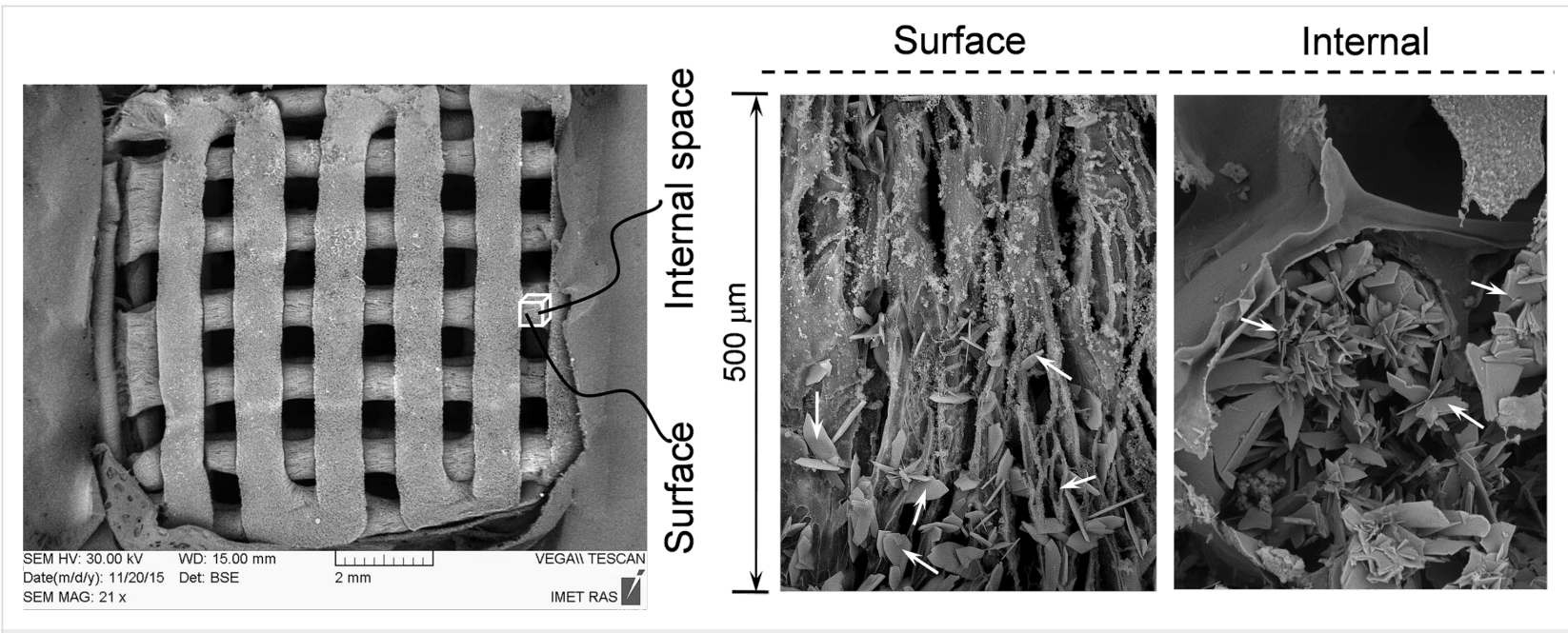

Figure 3: SEM images showing a general sample overview and details of the surface and internal region of a 3D printed sample (white arrows indicate DCPD crystals).

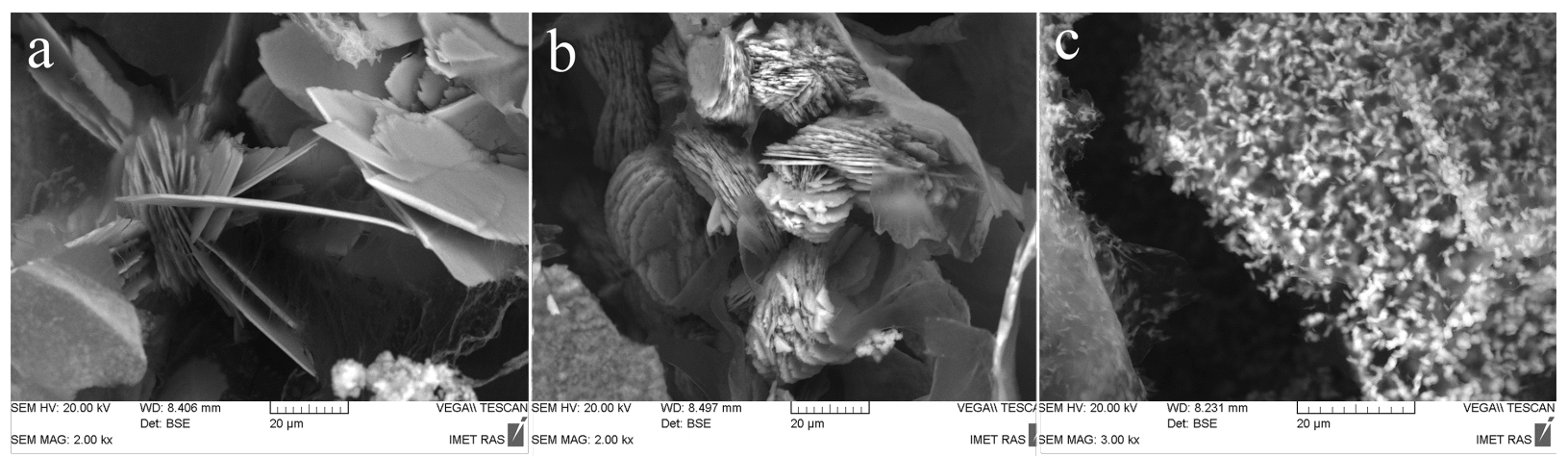

Figure 4: SEM micrographs of the microstructure of 3D printed samples on the basis of alginate with concentration of (a) 0.25 , (b) 1.0 and (c) $2.0 \mathrm{wt} \%$. 
tra of 3D printed DCPD-alginate samples, the bands are shifted from $1125 \mathrm{~cm}^{-1}$ for DCPD to $1114 \mathrm{~cm}^{-1}$ and from 1056 to $1064 \mathrm{~cm}^{-1}$. The bands at $981 \mathrm{~cm}^{-1}, 870 \mathrm{~cm}^{-1}, 575 \mathrm{~cm}^{-1}$ and $520 \mathrm{~cm}^{-1}$ shifted insignificantly (Figure 5 ). The $\mathrm{H}_{2} \mathrm{O}$ bands were observed to shift from 650 to $636 \mathrm{~cm}^{-1}$. Apparently, calcium phosphate reacts with alginate through the carboxyl groups of amino acids and calcium in calcium phosphate, because the frequency changes are observed for groups located near calcium ions in the phosphate structures [13].

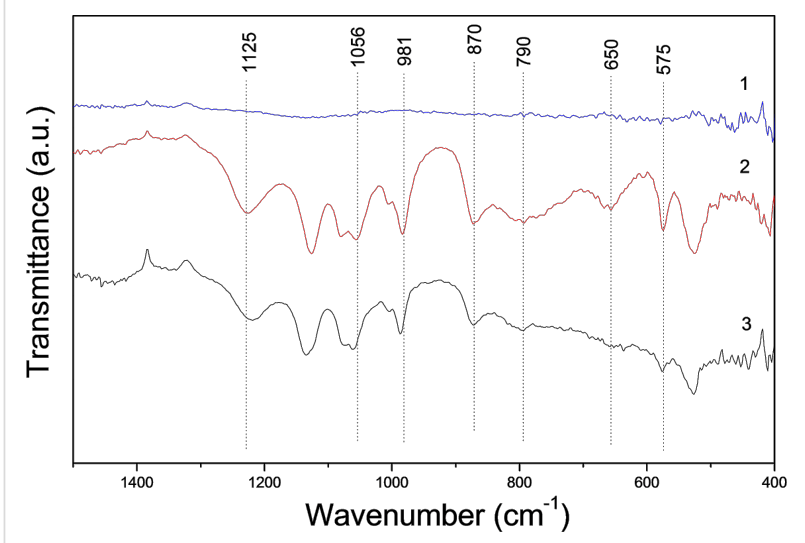

Figure 5: FTIR spectra of (1) sodium alginate, (2) DCPD, and (3) the 3D printed product.

The compressive strength of $3 \mathrm{D}$ printed samples is shown in Figure 6. The mechanical properties of 3D printed samples are relatively low due to the weak bonding between different printed layers. However, the compressive strength of composite materials increased with alginate concentration from $0.45 \mathrm{MPa}$ up to about $1.0 \mathrm{MPa}$ at $p \leq 0.005$. The increase in the compressive strength can be explained by an increase in alginate content within the 3D printed samples. Similar to our result it has been reported in the literature [16] that in situ precipitated calcium phosphate in polymeric composites tend to have lower mechanical strength.

The chemical and phase composition of the developed 3D printed samples can be adjusted further by chemical post-treatment. For instance, the hydrolysis of DCPD might lead to the development of an octacalcium phosphate phase and an adhesive effect between particles could take place [17].

\section{Conclusion}

We propose a new "biomimetic $+3 \mathrm{D}$ printing" approach for fabrication of complex-structured composite bone substitutes using appropriate raw materials and a new "ink". Depending on the processing parameters and conditions, it is possible to achieve materials with adjustable mechanical properties, speci-

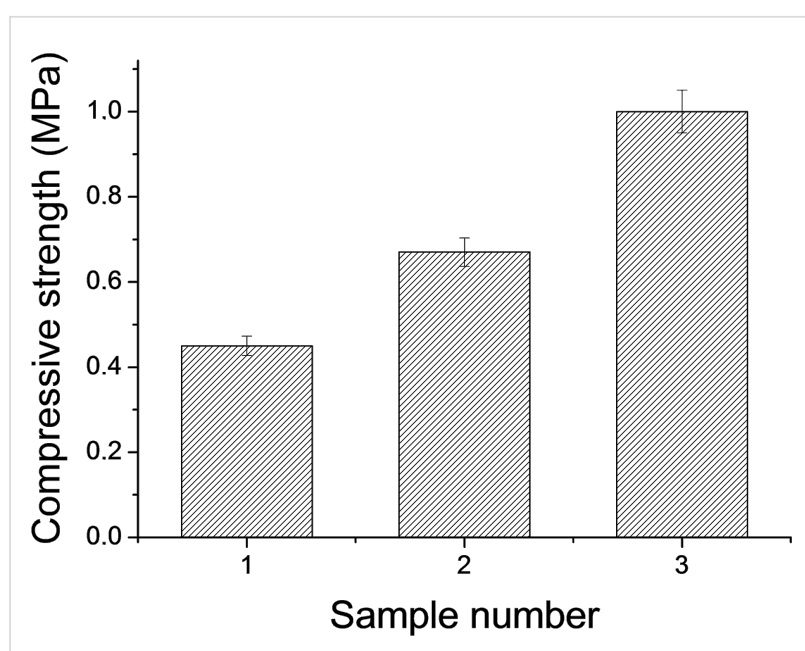

Figure 6: Compressive strength of 3D printed samples on the basis of alginate with concentration of (1) 0.25 , (2) 1.0 and (3) $2.0 \mathrm{wt} \%$.

fied composition, morphology and CP crystal size. Our results may provide a new approach for synthesis of highly osteogenic composite materials and for the effective fabrication of customdesigned implants and tissue engineering constructs via 3D printing on the commercial scale.

\section{Experimental \\ Materials}

The fabrication of 3D composite structures was performed using a binary system based on aqueous solutions of sodium alginate. The polymer slurry of sodium alginate (CAS number 9005-38-3) with concentrations of $0.25,1.0$ and $2.0 \mathrm{wt} \%$ was prepared in distilled water in presence of glutamic acid $\left(\mathrm{C}_{5} \mathrm{H}_{9} \mathrm{NO}_{4}\right)(\mathrm{CAS}$ number 56-86-0) with concentrations of 0.25 , 1.0 and $2.0 \mathrm{wt} \%$. Glutamic acid was used to maintain a low $\mathrm{pH}$ value. Then, a solution of ammonium hydrogen phosphate (CAS number 7783-28-0) with concentration of $5 \mathrm{wt} \%$ was added as the source of phosphorus in the system. The second "ink" was calcium chloride (CAS number 10043-52-4) water solution with a concentration of $10 \mathrm{wt} \%$, being the source of calcium. All reagents were purchased from Sigma-Aldrich.

\section{D printing}

A custom-designed 3D printer was used for our experiments, as shown in Figure 7. The initial reactant solutions were placed in two separate cartridges of 3D printer. Thereafter, they were injected through a nozzle (or needle, depending on the initial viscosity of the solutions) of disposable syringe on a cooled ( -5 to $-30{ }^{\circ} \mathrm{C}$ ) flat glass substrate. The $\mathrm{pH}$ of the reaction medium was measured by an Econix-Expert $001 \mathrm{pH}$ meter (EconixExpert Ltd., Moscow, Russia) and maintained at $\mathrm{pH} 4.5 \pm 0.5$. The printed samples were deep-frozen at $-50{ }^{\circ} \mathrm{C}$ and then freeze-dried at $6 \times 10^{-5} \mathrm{~atm}$ for 10-12 h. Finally, the fabricated 
$3 \mathrm{D}$ structure was washed in distilled water and incubated at $37{ }^{\circ} \mathrm{C}$ for one day.

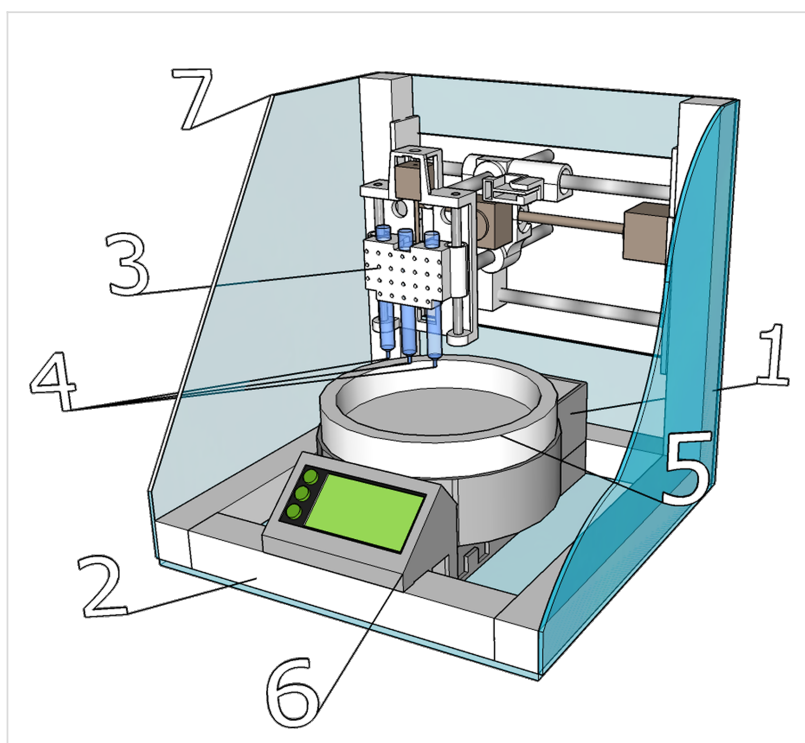

Figure 7: Custom-designed 3D printer. 1 - power supply; 2 - metal frame; 3 - printing head with a reservoir and heaters; 4 - nozzles; 5 - thermostatically controlled worktable; 6 - microcontroller unit and power electronic modules; 7 - sealed enclosure.

\section{Characterization}

As described in [17], the phase composition of the samples was analyzed by conventional X-ray diffraction (XRD) technique

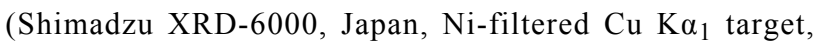
$\lambda=1.54183 \AA$ ). The samples were scanned at a $2 \theta$ angle from $10-60^{\circ}$ with a $0.02^{\circ}$ step and a preset time of $5 \mathrm{~s}$. A scanning electron microscopy apparatus (Tescan Vega II, Czech Republic), operated in secondary and backscattered electron modes, was used for 3D microstructure analysis. The samples were sputter-coated with a $25 \mathrm{~nm}$ thick gold layer prior to imaging, imparting electrical conductivity to the surfaces. FTIR spectroscopy (Nicolet Avatar 330, England) was performed after mixing $1 \mathrm{mg}$ of the grinded sample with $300 \mathrm{mg}$ of $\mathrm{KBr}$ powder followed by compacting into a thin pellet in a stainless steel die with a $1 \mathrm{~cm}$ inner diameter. FTIR data were recorded over the range of $4000-400 \mathrm{~cm}^{-1}$ with 128 scans.

As described in [17] the compressive strength of the samples was evaluated in accordance with the ISO standard 83.100: Cellular materials. Five samples for each point were used. Compression testing was carried out using an Instron 5581 (Bucks, UK) testing machine operating at a crosshead speed of $1 \mathrm{~mm} / \mathrm{min}$. Statistical analysis was performed using SPSS software, version 17.0 (Statistical Package for Social Sciences, SPSS Inc., USA). The mean and standard deviation of compressive strength were calculated.

\section{Acknowledgments}

This work was supported by the Russian Science Foundation (project No. 15-13-00108).

\section{References}

1. Kim, S. S.; Utsunomiya, H.; Koski, J. A.; Wu, B. M.; Cima, M. J.; Sohn, J.; Mukai, K.; Griffith, L. G.; Vacanti, J. P. Ann. Surg. 1998, 228, 8-13.

2. Bose, S.; Vahabzadeh, S.; Bandyopadhyay, A. Mater. Today 2013, 16, 496-504. doi:10.1016/j.mattod.2013.11.017

3. Chia, H. N.; Wu, B. M. J. Biol. Eng. 2015, 9, 1-14.

4. Wang, X.; Schröder, H. C.; Müller, W. E. G. Beilstein J. Nanotechnol. 2014, 5, 610-621. doi:10.3762/bjnano.5.72

5. Rees, D. A. Pure Appl. Chem. 1981, 53, 1-14. doi:10.1351/pac198153010001

6. Li, Y.; Liu, Y.; Li, S.; Liang, G.; Jiang, C.; Hu, Q. J. Biosci. Bioeng. 2016, 121, 111-116. doi:10.1016/j.jbiosc.2015.04.020

7. Oliveira de Lima, D.; Aimoli, C. G.; Beppu, M. M. Mat. Sci. Eng. C 2009, 29, 1109-1113. doi:10.1016/j.msec.2008.09.019

8. Dorozhkin, S. V.; Epple, M. Angew. Chem., Int. Ed. 2002, 41, 3130-3146. doi:10.1002/1521-3773(20020902)41:17<3130::AID-ANIE3130>3.0.CO ;2-1

9. Shor, L.; Güçeri, S.; Wen, X.; Gandhi, M.; Sun, W. Biomaterials 2007, 28, 5291-5297. doi:10.1016/j.biomaterials.2007.08.018

10. Serra, T.; Planell, J. A.; Navarro, M. Acta Biomater. 2013, 9 , 5521-5530. doi:10.1016/j.actbio.2012.10.041

11. Inzana, J. A.; Olvera, D.; Fuller, S. M.; Kelly, J. P.; Graeve, O. A.; Schwarz, E. M.; Kates, S. L.; Awad, H. A. Biomaterials 2014, 35, 4026-4034. doi:10.1016/j.biomaterials.2014.01.064

12. Calvert, P.; Rieke, P. Chem. Mater. 1996, 8, 1715-1727. doi:10.1021/cm960126o

13. Amer, W.; Abdelouahdi, K.; Ramananarivo, H. R.; Fihri, A.; El Achaby, M.; Zahouily, M.; Barakat, A.; Djessas, K.; Clark, J.; Solhy, A. Mat. Sci. Eng. C 2014, 35, 341-346. doi:10.1016/j.msec.2013.11.012

14. Davis, T. A.; Volesky, B.; Mucci, A. Water Res. 2003, 37, 4311-4330. doi:10.1016/S0043-1354(03)00293-8

15. Xu, J.; Butler, I. S.; Gilson, D. F. R. Spectrochim. Acta, Part A 1999, 55, 2801-2809. doi:10.1016/S1386-1425(99)00090-6

16. Jin, H.-H.; Lee, C.-H.; Lee, W.-K.; Lee, J.-K.; Park, H.-C.; Yoon, S.-Y. Mater. Lett. 2008, 62, 1630-1633. doi:10.1016/j.matlet.2007.09.043

17. Komlev, V. S.; Popov, V. K.; Mironov, A. V.; Fedotov, A. Yu.; Teterina, A. Yu.; Smirnov, I. V.; Bozo, I. Y.; Rybko, V. A.; Deev, R. V. Front. Bioeng. Biotechnol. 2015, 3, No. 81. doi:10.3389/fbioe.2015.00081 


\section{License and Terms}

This is an Open Access article under the terms of the Creative Commons Attribution License

(http://creativecommons.org/licenses/by/4.0), which permits unrestricted use, distribution, and reproduction in any medium, provided the original work is properly cited.

The license is subject to the Beilstein Journal of Nanotechnology terms and conditions:

(http://www.beilstein-journals.org/bjnano)

The definitive version of this article is the electronic one which can be found at:

doi:10.3762/bjnano.7.172 